\title{
Measuring the Factors of Hesitancy in Saudi Population toward COVID-19 Vaccines
}

\author{
Dr. Roba Attar ${ }^{1 *}$, Dr. Alaa Karkashan ${ }^{1}$, Dr. Basma Abbas ${ }^{1}$, Mr. Abdullatif Almarashi², \\ Mr. Talat Hashem ${ }^{3}$. \\ 1 Department of Biology, University of Jeddah, \\ ${ }^{2}$ Research and Studies department, Jeddah Province, Ministry of health, Saudi Arabia \\ ${ }^{3}$ Makkah Regional laboratory, Ministry of health, Saudi Arabia \\ Email: rmattar@uj.edu.sa
}

\begin{abstract}
COVID-19 has major effects on the population, enforcing lockdowns and strict precautions across the world to deter the virus from spreading. The pandemic presents a significant threat to our health and well-being. As vaccines become available, COVID-19 lethality may be reduced by promoting widespread immunization. To achieve herd immunity thresholds for COVID-19, an estimated $70 \%$ of the population must be vaccinated. The public's approval determines the success of the vaccination program. Understanding the factors that contribute to COVID-19 vaccine hesitancy is important. Therefore, this cross-sectional study was conducted on the Saudi population from all the regions of the Kingdom of Saudi Arabia to evaluate the level of knowledge about COVID-19 vaccines, estimate the turnout level for receiving the COVID-19 vaccine, and understand the reasons behind hesitancy toward COVID-19 vaccines. A total of 1,148 adults completed a web-based questionnaire, and the study tested several sociocultural and environmental variables that affect the community's hesitancy toward vaccination. Results showed that gender characteristics had almost no effect on acceptance of the vaccination. Individuals of the younger age group $<30$ demonstrated an increase in the rate of vaccine hesitancy (53\%) compared with individuals from the older age groups $>40(34.43 \%)$.
\end{abstract}


In addition, middle and higher education groups were found to have significant vaccine hesitancy $(77.4 \%)$ compared with the less educated group $(41.1 \%)$. The most common concern among the non-vaccine takers in this questionnaire was that of vaccine safety: these participants believed the vaccine would result in health problems (49.3\%), and most participants agreed (64\%) that this was due to the insufficient duration of vaccine administration for safety evaluation.

Keywords: COVID-19; Hesitancy; Acceptance; Saudi Arabia; Vaccine

\section{Introduction}

In late December 2019, a serious acute respiratory disease outbreak, attributed to a new strain of coronavirus (COVID-19), occurred in Wuhan, China (Poland, 2020). The illness spectrum of COVID-19 is broad, ranging from asymptomatic infection to acute respiratory distress syndrome, resulting in death ("NIH, COVID-19 Treatment Guidelines", 2021). In response to the pandemic, dozens of vaccine research efforts have been launched (Kaur \& Gupta, 2020).

The COVID-19 pandemic has had a major effect on populations, with governments across the world enforcing travel limits and other interventions such as obligatory face coverings or quarantine to deter the virus from spreading (Sherman et al., 2020). The world is facing a novel infectious illness as a result of the COVID-19 pandemic, which presents a significant threat to our health and well-being ("WHO Director-General's opening remarks at the media briefing on COVID-19-11 March 2020", 2021). COVID-19's lethality should promote widespread immunization as a vaccine becomes available. Unfortunately, recent public polling data in the United States reported otherwise, with $20-27 \%$ of the public claiming that they would refuse to be vaccinated against the infection ("Most Americans expect a COVID-19 vaccine within a year; $72 \%$ say they would get vaccinated", 2021).

Moderna and Pfizer-BioNTech reported their results from randomized trials of COVID-19 vaccines in November 2020, revealing an impressive 90-95\% efficacy (Polack et al., 2020). On December 10, 2020, the Food and Drug Administration (FDA) released Emergency Use Authorizations (EUA) for the Pfizer-BioNTech vaccine, and on December 18, 2020, for the Moderna vaccine ("Coronavirus (COVID-19) Update: FDA Holds Advisory Committee Meeting to Discuss Authorization of COVID-19 Vaccine Candidate as Part of Agency's Review of Safety 
and Effectiveness Data", 202; "FDA Takes Additional Action in Fight Against COVID-19 By Issuing Emergency Use Authorization for Second COVID-19 Vaccine", 2021). To achieve herd immunity thresholds for COVID-19, an estimated $70 \%$ of the population must be vaccinated (Fine et al., 2011; Kwok et al., 2020; Weinberg \& Szilagyi, 2010). The vaccination program's success will be determined by the public's approval of the vaccines. Understanding the causes that contribute to COVID-19 vaccine hesitancy is important. To dispel people's skepticism about such vaccinations, strong public health programs must be created and continued. Effective public communication necessitates formative research into the awareness gaps, expectations, values, health behaviors, and demographic characteristics that contribute to anticipated COVID-19 vaccine hesitancy (Ruiz \& Bell, 2021).

The effective production and universal acceptance of a SARS-CoV-2 vaccine would be a significant step in combating the pandemic, but achieving high uptake will be difficult, exacerbated by online disinformation (Loomba et al., 2021). Conventional and social media play a role in the production of COVID-19 vaccine hesitancy. Understanding that people are suspicious of a COVID-19 vaccine is critical because this will help health officials increase vaccine awareness and thereby reduce disease transmission. It is estimated that to achieve herd immunity, a novel COVID-19 vaccine must be accepted by at least 55\% of the population (Kwok et al., 2020; Sanche et al., 2020).

If large-scale vaccine rejection threatens herd immunity goals, large-scale acceptance of local vaccine rejection may also have significant consequences for community (herd) immunity because clustering of non-vaccinators can disproportionately increase the required percentage of vaccination coverage to achieve herd immunity in adjacent geographical regions and encourage epidemic spread (Salathé \& Bonhoeffer, 2008). While studies have been conducted to investigate the impact of misinformation about COVID-19 on public perceptions of the pandemic (Geldsetzer, 2020; Islam et al., 2020; Kim et al., 2020) and the proclivity of some socio-political groups to believe misinformation (Kreps \& Kriner, 2020; Murphy et al., 2021), experts still lack a quantitative understanding of the relationship between exposure to misinformation about COVID19 and intent to receive the vaccination. 
If a viable vaccine becomes available, it is critical to consider this relationship, how it affects various sociodemographic groups, and whether groups at high risk of experiencing serious COVID-19 complications are vulnerable to misinformation (Loomba et al., 2021).

Several studies on the decision to obtain the vaccine were conducted during the vaccine production phase. Moreover, various studies have revealed many factors that influence vaccine acceptability when a new vaccine is launched (Gidengil et al., 2012; Larson et al., 2018; Setbon \& Raude, 2010; Xiao \& Wong, 2020). These factors include the vaccine's safety and potency, adverse health effects, misconceptions regarding the need for vaccination, a lack of confidence in the health system, and a lack of general awareness about vaccine-preventable diseases (Setbon \& Raude, 2010; Halpin \& Reid, 2019).

Vaccine hesitancy is an obstacle to the effectiveness of immunization programs and has been listed by the World Health Organization (WHO) as one of the top 10 global health challenges in 2019 ("Ten health issues WHO will tackle this year", 2021). A comprehensive understanding of current vaccine attitudes and potential determinants of people's behavior is essential for designing effective health communications to encourage uptake and implement successful demographic immunization. Vaccine hesitancy is a serious threat in the struggle against COVID-19 because achieving herd immunity is dependent on the efficacy of the vaccine and the community's willingness to accept it (Lazarus et al., 2020). The multifaceted nature of vaccine hesitancy, including willingness to accept COVID-19 vaccines, can complicate studies into its global effects (MacDonald, 2015).

Despite significant attempts to develop effective COVID-19 vaccines, a major impediment may be attributed to vaccine hesitancy against accepted and prospective COVID-19 vaccination (Harrison \& Wu, 2020). Many studies about whether or not to take the vaccine were conducted prior to the vaccine's availability. Statistics suggest that the vaccine's adoption is gradually increasing. A total of 1,448,242,899 vaccine doses had been delivered as of May 20, 2021. According to UN data, the 2020 population in Saudi Arabia is expected to be $34,813,871$ people at the mid-year point, yet just 15,099,896 vaccine doses have been administered ("WHO Coronavirus Disease (COVID-19) Dashboard", 2020).

This cross-sectional study was conducted among the Saudi population to assess the level of knowledge about the COVID-19 vaccine, 
Estimate the level of participation in the COVID-19 vaccine, and understand the reasons for hesitancy and fear about the COVID-19 vaccines to determine the scope of the problem.

\section{Material and Methods}

The study design was cross-sectional, and we used the Google Docs platform to design and disseminate a questionnaire online through social media applications (Twitter and WhatsApp) to achieve snowball sampling with participants from all over the country. We distributed the questionnaire and collected responses within only three days to avoid bias that may occur because beliefs and behaviors change over time.

\subsection{Sample Size}

The population of the Kingdom of Saudi Arabia is around 35,013,414 million, according to the latest population census in 2020 ("Reports and statistics", 2021). Moreover, according to the Assistant Deputy Minister for Preventive Health, Dr Abdullah Assiri, the ministry plans to vaccinate 26 million people ( $88 \%$ of the population) by the end of 2021 (Assiri, 2021). We entered these numbers into Epi Info 7 software and estimated a sample size of 969 participants at $80 \%$ power and $99.99 \%$ confidence interval. However, we collected 1,148 responses to be able to exclude incomplete questionnaires.

\subsection{Study Tool}

The questionnaire was designed after reviewing similar studies that had been conducted in other countries. The authors of the current paper held an online meeting to discuss these studies and choose the most relevant questions, which were customized for the context of the local culture. After agreeing on the questionnaire, a well-constructed online questionnaire was disseminated using the Google Docs platform. The questionnaire consisted of two parts: one to gather sociodemographic data, and another to assess criteria such as age, education, and work in relation to the level of awareness about the COVID-19 vaccine. 


\section{3. $\quad$ Ethical Considerations}

The study was ethically approved by the ethics committee of Jeddah Health Affairs before starting data collection. In the first part of the questionnaire, we identified ourselves and stated the participants' rights. At the end of the questionnaire, we informed participants that an email address would be provided should participants have any inquiries regarding unclear questions or should they wish to withdraw from participation. Additionally, participants were informed that no names would be recorded on the questionnaires and that all personal information about participants would be kept confidential.

\section{Results}

According to the study design, 1,148 adult individuals from all regions in the Kingdom of Saudi Arabia responded to the electronic questionnaire describing their experiences with the COVID-19 vaccine. The results section comprises two main sections: the first describes the characteristics of the participants and their experience regarding the vaccine; the second presents the factors potentially associated with disagreement about receiving the vaccine.

\subsection{Characteristics of the Study Group and Their Experience of the COVID-19 Vaccine}

There was a slight dominance of females $(59.3 \%)$ over males $(40.7 \%)$, and the vast majority were Saudis $(92.2 \%)$. The mean age of the participants accounted for $36.9 \pm 8.7$ years, about onethird (34.2\%) being 30-39 years and slightly more than one-third (37.9\%) being 40 years or older. Most of the participants possessed either a bachelor's qualification (56.6\%) or a postgraduate degree $(28.8 \%)$. Only one-third of the participants $(34.4 \%)$ were either jobless or still students, and the remainder had jobs mainly in the health sector (20.6\%) and the education sector (Table 1).

The majority of the participants $(70.2 \%)$ reported that they had been tested for COVID-19 before, and $12.1 \%$ had tested positive for COVID-19. Most of the positive cases $(86.3 \%)$ were advised to isolate themselves at their homes, while $13.7 \%$ were referred to quarantine isolation (Table 2). 
When asked to rate the seriousness of the COVID-19 disease, less than one-half of participants $(46.1 \%)$ regarded it as a very serious disease, $44.7 \%$ stated that it is not a serious disease, while $6.9 \%$ claimed that it is not serious at all (Figure 1).

Table 2 shows that most of the participants (72.7\%) thought that the COVID-19 vaccine is important, and $68.0 \%$ had received it. Among those who had received the vaccine, besides believing that the vaccine is important, fear of catching the disease $(38.7 \%)$ and benefits from the merits of being vaccinated (36.5\%) were the main impetus for seeking vaccination. The Pfizer vaccine was the most commonly received (63.9\%), while AstraZeneca was given to 34.8\%, which is nearly in line with participants' preferences for type of vaccine, with $62.1 \%$ preferring the Pfizer vaccine. The reasons for preferences for certain types of vaccine ranged between scientific issues $(64.6 \%)$, social effects $(31.8 \%)$, and media effects $(53.4 \%)$. The overwhelming majority of those who had received the vaccine $(87.1 \%)$ stressed that they would advise others to take it.

\subsection{Vaccine Non-takers and Factors Potentially Associated with Disagreeing to Take the Vaccine.}

Only one-quarter of those who did not receive the vaccine $(26.4 \%)$ thought that the vaccine is safe, and one-half $(50.4 \%)$ claimed that they had no idea about the safety of the vaccine. The main reason for not taking the vaccine was fear of side effects $(42.5 \%)$, and the main sources for information about the vaccine were media web pages (32.2\%), international media (27.8\%), and social media (19.6\%). One-half of the vaccine non-takers believed that the vaccine would be harmful $(49.3 \%)$. They attributed their belief to the insufficient duration of vaccine testing (64\%) and scarce information about it (51.2\%), while $14.2 \%$ believed that there is a conspiracy behind the administration of the vaccine (Table 4 ). Figure 2 demonstrates that $42 \%$ of vaccine non-takers would agree to receive it, while $58 \%$ would refuse it. The following section describes factors potentially associated with disagreeing to take the vaccine.

Table 5 demonstrates that the younger the age, the more likely the agreement to take the vaccine; the percentage of those who agreed ranged from 53\% among those aged $<30$ years to $34.3 \%$ among those aged 40 years or older. Also, agreement was significantly higher in those with lower education levels $(55.9 \%)$ compared with those with a bachelor qualification (38.9\%) or a postgraduate degree $(38.5 \%)(\mathrm{p}<0.05)$. 
Although agreement was higher among males (46.9\%), non-Saudis (52.4\%), the jobless and students $(46.9 \%)$, these differences are not statistically significant $(\mathrm{p}>0.05)$.

Table 6 shows that those who believed COVID-19 is a serious disease were significantly more likely to agree to receive the vaccine (47.3\%) than those who did not believe it is (18.9\%) (p $<0.05)$. In addition, those who perceived that vaccination would return life to normal were significantly more likely to agree to receive the vaccine $(64.1 \%)$ than were those who did not perceive this $(14.8 \%)(\mathrm{p}<0.05)$. Conversely, those who perceived that the vaccine would be harmful were significantly less likely to agree to vaccination $(19.3 \%)$ than those who thought that it is not harmful $(68 \%)(\mathrm{p}<0.05)$.

Table 7 provides the backwards Wald regression model for factors predicting disagreeing to take the vaccine. The model shows that those who did not believe the vaccine would help return life to normal were nearly six times more likely to refuse to take the vaccine than those who believed it would return life to normal $(\mathrm{p}<0.005)$. The second predictor for disagreement was participants' perception of the harmful effects of the vaccine, for which those who did not regard it as harmful were negatively associated with refusal to take the vaccine $(\mathrm{p}<0.05)$.

Table 1: Sociodemographic characteristics of the study group $(n=1,148)$.

Characteristics

\section{Gender}

Male

Female

Nationality

Saudi

Non-Saudi

\section{Age categories}

$<30$ years

30-39 years

$\geq 40$ years

435

\section{Education level}

Lower than secondary

Secondary

Bachelor
40.7

59.3

\section{1}

92.2

90

7.8 
Academic Journal of Research and Scientific Publishing | Vol 3 | Issue 29

Publication Date: 5-9-2021 ISSN: 2706-6495

Postgraduate

331

28.8

\section{Job/field}

Jobless/student

395

34.4

Has a job

753

65.6

Health sector

237

20.6

Education sector

163

14.2

Military field

30

2.6

Administrative field

Engineering field

54

4.7

Other fields

112

9.8

Table 2: Previous testing and exposure to COVID-19 (n = 1,148).

Testing and exposure to COVID-19

No.

Percentage

Previously tested for COVID-19

Yes

806

70.2

No

342

29.8

Previously diagnosed with COVID-19

Yes

No

1,009

87.9

Measures taken after being diagnosed with COVID-19 (n

= 139)

Home isolation advised

120

86.3

Referred to quarantine isolation

19

13.7 
Academic Journal of Research and Scientific Publishing | Vol 3 | Issue 29

Publication Date: 5-9-2021 ISSN: 2706-6495

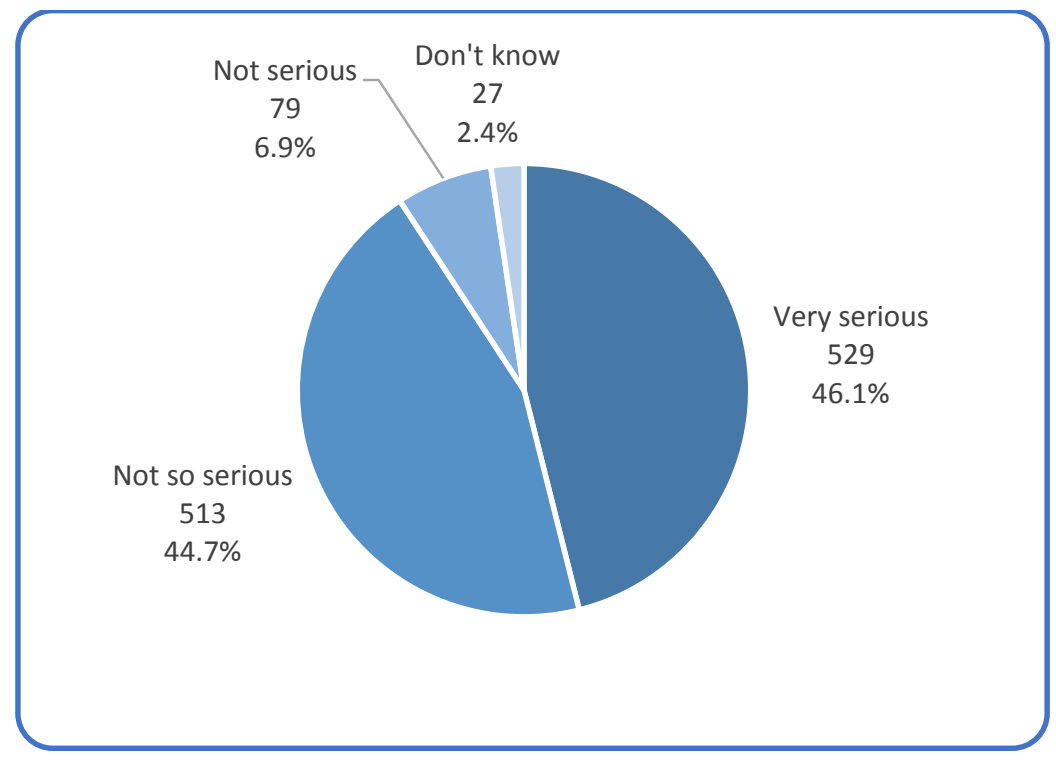

Figure 1: Rating of participants' beliefs about seriousness of COVID-19.

Table 3: Participants' experience of the COVID-19 vaccine.

\begin{tabular}{lcc}
\hline \hline & No. & Percentage \\
\hline Think that COVID-19 vaccine is important & 835 & 72.7 \\
Yes & 139 & 12.1 \\
No & 174 & 15.2 \\
Don't know & & \\
\hline Received the vaccine & 781 & 68.0 \\
Yes & 367 & 32.0 \\
No & 510 & 65.3 \\
\hline Impetus for seeking vaccine $(\boldsymbol{n}=\mathbf{7 8 1})$ & 303 & 38.7 \\
Believe that the vaccine is important & 106 & 13.6 \\
Fear of catching the disease & 34 & 4.4 \\
Influence of family and friends & 285 & 36.5 \\
Obligated by employment & 74 & 9.5 \\
Received merits for being immunized & & \\
Other & 499 & 63.9 \\
\hline Type of received vaccine $(\boldsymbol{n}=\mathbf{7 8 1})$ & 272 & 34.8 \\
Pfizer & & \\
AstraZeneca & & \\
\hline www.ajrsp.com & & \\
\hline
\end{tabular}


Academic Journal of Research and Scientific Publishing | Vol 3 | Issue 29

Publication Date: 5-9-2021 ISSN: 2706-6495

Don't know

9

1.3

Preference for certain vaccine $(n=781)$

Yes, preferred Pfizer vaccine 485

62.1

Yes, preferred AstraZeneca vaccine

No

272

34.8

Reasons for preferring certain vaccine $(n=509)$

Scientific issues

64.6

Social effect

Media effect

272

53.4

Would advise others to receive the vaccine $(n=781)$

Yes

680

87.1

No

Not sure

94

12.0

Table 4: Opinions and knowledge of vaccine skeptics regarding the COVID-19 vaccine $(\mathbf{n}=367)$.

No.

Percentage

\section{Think that the vaccine is safe}

Yes

85

26.4

No

185

23.2

Don't know

\section{Reasons for not taking the vaccine}

Suffer from health problems 38

10.4

There is no good reason to take it

24.8

Fear of side effects

156

42.5

Influence of friends

Influence of social media

54

14.7

No particular reason

110

30.0

\section{Sources of information about the vaccine}

Local media

International media

102 
Academic Journal of Research and Scientific Publishing | Vol 3 | Issue 29

Publication Date: 5-9-2021 ISSN: 2706-6495

\begin{tabular}{lcc}
\hline \hline Local news sites & 25 & 6.8 \\
Medical web pages & 118 & 32.2 \\
Social media & 67 & 18.3 \\
Family and friends & 72 & 19.6 \\
Others & 207 & 56.4 \\
\hline Think that the vaccine could result in harmful health & & \\
problems & 181 & 49.3 \\
Yes & 50 & 13.6 \\
No & 136 & 37.1 \\
Don't know & & \\
\hline Reasons to think that vaccine could be harmful & 235 & 64.0 \\
The duration was not sufficient to test the vaccine well & 188 & 51.2 \\
There is not enough information about the vaccine & 52 & 14.2 \\
Believe that there is a conspiracy behind the vaccine & & \\
\hline \hline
\end{tabular}

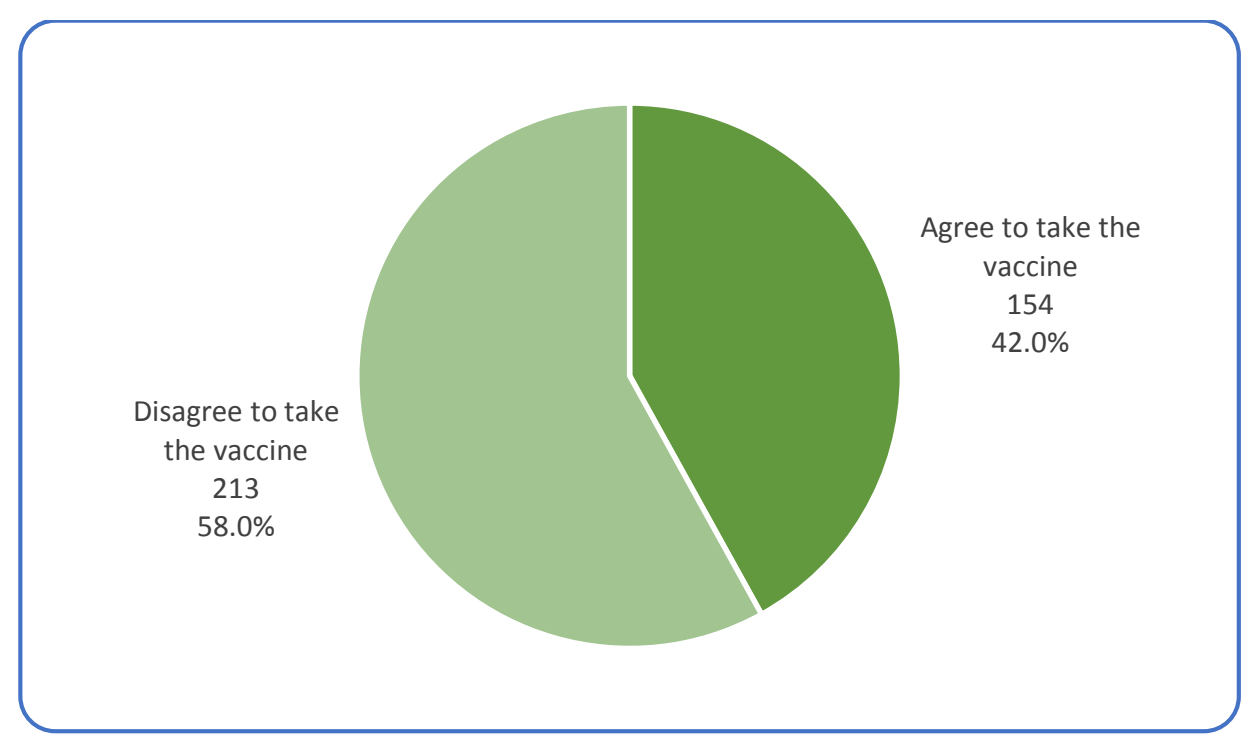

Figure 2: Agreement of the unvaccinated participants to take the vaccine. 
Academic Journal of Research and Scientific Publishing | Vol 3 | Issue 29

Publication Date: 5-9-2021 ISSN: 2706-6495

Table 5: Agreement of the unvaccinated respondents to take the vaccine according to their characteristics $(n=367)$.

\begin{tabular}{llll}
\hline \multirow{3}{*}{ Variables } & \multicolumn{4}{c}{ Agreement to take the vaccine } \\
\cline { 2 - 4 } & \multicolumn{2}{c}{ Agree } & Disagree
\end{tabular}$X^{2} \quad \mathrm{P}^{*}$

\section{Gender}

\begin{tabular}{lcccccc} 
Male & 53 & $46.9 \%$ & 60 & $53.1 \%$ & & \\
Female & 101 & $39.8 \%$ & 153 & $60.2 \%$ & 1.637 & 0.201 \\
\hline $\begin{array}{l}\text { Nationality } \\
\text { Saudi }\end{array}$ & 132 & $40.6 \%$ & 193 & $59.4 \%$ & & \\
Non-Saudi & 22 & $52.4 \%$ & 20 & $47.6 \%$ & 2.114 & 0.146 \\
\hline
\end{tabular}

\section{Age categories}

$\begin{array}{lllllll}<30 \text { years } & 70 & 53.0 \% & 62 & 47.0 \% & & \\ 30-<40 \text { years } & 48 & 36.9 \% & 82 & 63.1 \% & 10.356 & 0.005 \\ \geq 40 \text { years } & 36 & 34.3 \% & 69 & 65.7 \% & & \end{array}$

\section{Education level}

\begin{tabular}{lcccccc} 
Secondary or lower & 38 & $55.9 \%$ & 30 & $44.1 \%$ & & \\
Bachelor & 79 & $38.9 \%$ & 124 & $61.1 \%$ & 6.645 & 0.036 \\
Postgraduate & 37 & $38.5 \%$ & 59 & $61.5 \%$ & & \\
\hline
\end{tabular}

\section{Job}

\begin{tabular}{lcccccc} 
Jobless/student & 76 & $46.9 \%$ & 86 & $53.1 \%$ & & \\
Has a job & 78 & $38.0 \%$ & 127 & $62.0 \%$ & 2.920 & 0.087 \\
\hline
\end{tabular}

* Based on chi-square.

Table 6: Perceptions potentially associated with agreement to receive COVID-19 vaccine.

\begin{tabular}{|c|c|c|c|c|c|c|}
\hline \multirow{3}{*}{ Variables } & \multicolumn{4}{|c|}{ Agreement to take the vaccine } & \multirow{3}{*}{$\mathrm{X} 2$} & \multirow{3}{*}{$\mathrm{P}^{*}$} \\
\hline & \multicolumn{2}{|c|}{ Agree } & \multicolumn{2}{|c|}{ Disagree } & & \\
\hline & No & $\%$ & No & $\%$ & & \\
\hline
\end{tabular}

Think that COVID-19 is

a serious disease

$\begin{array}{lllllll}\text { Yes, very serious } & 62 & 47.3 \% & 69 & 52.7 \% & 14.354 & 0.002\end{array}$


Academic Journal of Research and Scientific Publishing | Vol 3 | Issue 29

Publication Date: 5-9-2021 ISSN: 2706-6495

\begin{tabular}{lcccccc} 
Yes, not so serious & 76 & $44.7 \%$ & 94 & $55.3 \%$ & & \\
Not serious & 10 & $18.9 \%$ & 43 & $81.1 \%$ & & \\
Don't know & 4 & $30.8 \%$ & 9 & $69.2 \%$ & & \\
\hline $\begin{array}{l}\text { Think that the vaccine } \\
\text { could be harmful }\end{array}$ & & & & & & \\
Yes & 35 & $19.3 \%$ & 146 & $80.7 \%$ & & \\
No & 34 & $68.0 \%$ & 16 & $32.0 \%$ & 72.491 & $<0.001$ \\
Don't know & 83 & $61.0 \%$ & 53 & $39.0 \%$ & & \\
\hline $\begin{array}{l}\text { Think that the vaccine } \\
\text { could help return to }\end{array}$ & & & & & & \\
normal life & & & & & & \\
Yes & 98 & $64.1 \%$ & 55 & $35.9 \%$ & & \\
No & 17 & $14.8 \%$ & 98 & $85.2 \%$ & & \\
Don't know & 37 & $37.4 \%$ & 62 & $62.6 \%$ & & \\
\hline \hline
\end{tabular}

* Based on chi-square.

Table 7: Factors predicting disagreement to take COVID-19 vaccine.

\begin{tabular}{|l|c|c|c|c|c|c|}
\hline & $\mathrm{B}$ & S.E. & Wald & $\mathrm{df}$ & Sig. & Exp(B) \\
\hline Returns life to normal & & & & & & \\
Yes & & & 32.738 & 2 & .000 & \\
No & 1.793 & .338 & 28.094 & 1 & .000 & 6.007 \\
Don't know & 1.200 & .312 & 14.751 & 1 & .000 & 3.319 \\
Think the vaccine is & & & & & & \\
harmful & & & & & & \\
Yes & & & 41.425 & 2 & .000 & \\
No & 1.604 & .388 & 17.081 & 1 & .000 & .201 \\
Don't know & 1.751 & .289 & 36.569 & 1 & .000 & .174 \\
Constant & .048 & .363 & .017 & 1 & .895 & .953 \\
\hline
\end{tabular}

Note. Factors excluded from the final model for non-significance: age, education level, seriousness of COVID-19 disease 


\section{Discussion}

For decades, vaccines have proven effective in eradicating several deadly diseases, such as smallpox and measles (Al-Mohaithef \& Padhi, 2020). In light of the emergence of the COVID19 virus, which has claimed the lives of many, most of the world's eyes are directed toward the leading companies in the field of vaccines to produce a vaccine that saves people from the dreaded COVID-19 virus (Harrison \& Wu, 2020). In contrast, a sizable number of people are unsure about the effects of the COVID-19 vaccination. Vaccine hesitancy has been a known phenomenon since the advent of the vaccine revolution, and it has had a significant impact on disease control (Benecke \& DeYoung, 2019; Phadke et al., 2016).

This study included 1,148 adults from all regions of the Kingdom of Saudi Arabia who completed a web-based questionnaire to share their experiences regarding the COVID-19 vaccine (Lazarus et al., 2020). Several socio-cultural and environmental variables that affect a community's vaccination hesitancy were tested, such as gender, age, education, and media influence (Cordina et al., 2021; Qunaibi et al., 2021).

It is evident that gender characteristics had almost no effect on vaccination hesitancy, although the proportion of female respondents was relatively higher than male respondents.

This finding contradicts studies that have shown a higher tangible effect on vaccine hesitancy in women than in men (Fisher et al., 2020; Khubchandani et al., 2020). In contrast, men are less likely than women to engage in preventive behavior (Cordina et al., 2021).

The age characteristic showed a clear increase in the rate of vaccine acceptance for individuals of the younger age group $(<30)$ by $53 \%$, compared with $34.43 \%$ for individuals from the older age groups (> 40), and this finding differs from the results of Szilagyi, which states that the age group of fewer than 40 years appears to demonstrate significant hesitation towards the vaccine, while the age group over the age of 60 shows a desire to receive the vaccine (Loomba et al., 202; Szilagyi et al., 2021).

Interestingly, educational background had a negative effect on vaccine frequency, with $77.4 \%$ of those with middle or higher education expressing vaccine hesitancy, compared to $41.1 \%$ of those with lower education. These results are consistent with the results of the Loomba study conducted in the United Kingdom and the United States of America (Sherman et al., 2020; Loomba et al., 202). 
Nonetheless, in a study conducted in Guyana, the results were contradictory: the educated group was the least hesitant about the COVID-19 vaccine, while the less educated group was not only hesitant to take the COVID 19 vaccine but also recommended others around them not to be vaccinated (UNICEF, 2021).

In our study, we analyzed the relationship between vaccination hesitancy and the use of social media and official websites to become informed about the pandemic. We discovered that $32.2 \%$ of the information came from media web pages, $27.8 \%$ from international media and $19.6 \%$ from social media. Some researchers believe that this difference may be due to several reasons, including, for example, the level of trust in science and scientists, easy access to publications related to negative outcomes of COVID-19 vaccines, or the influence of social media and local and international news, all of which have a clear effect on hesitancy toward COVID-19 vaccines (Cordina et al., 2021; Biswas et al., 2021).

In this context, vaccine type preference was evident when the Pfizer vaccine scored $63.9 \%$ versus AstraZeneca's 34.8\%. Regardless of the scientific background of how these vaccines work, the administration of the AstraZeneca vaccine has received a blow because of the vaccine's potential side effects: namely, the development of thromboembolism, which has led to the death of a very few people who received the AstraZeneca vaccine. This was published by the local and international media, with a serious negative effect on the vaccine's uptake (Wise, 2021). Further, a number of European countries halted the AstraZeneca vaccine, which has led people in the Kingdom of Saudi Arabia to feel less skeptical of the Pfizer vaccine (Østergaard et al., 2021).

\section{Conclusion}

In this study, we explored people's response at various ages, education levels, and genders to the COVID-19 vaccine. However, vaccine hesitancy cannot be presented in this study because it is not a qualitative or quantitative study; rather, it is a scoping study that provides us with a general orientation towards Saudi society's opinion of the COVID-19 vaccine.

\section{Recommendations}

The study clearly shows that vaccine hesitancy among older people is higher compared to younger people. 
Based on this surprising result, we suggest that decision makers should work on broadcasting reassurance messages dedicated to the elderly about the importance of the vaccine and not having a negative effect on them. Moreover, people with higher education degrees tend to wait to accept the vaccine, waiting for more evidence about its effectiveness and the absence of side effects. Local and reliable media should allocate large space in their coverage to covering and translating COVID-19 Vaccine studies to provide more evidence that encourages educated people to make the decision to take the vaccine.

\section{Source of funding}

This research received no specific grant from any funding agency in the public, commercial, or not-for-profit sectors.

\section{Conflict of interest}

The authors have no conflict of interest to declare.

\section{Ethical approval}

This study was ethically finally approved by the Local Committee for Research Ethics in Jeddah health affairs on 15/6/2021 with the approval number: A01128.

\section{Author's contributions}

RA and AK conceptualized the study, drafted the questionnaire, conducted research, and collected and organized the data. RA wrote the initial and final draft of the introduction, accountable for all aspects of the work. AK wrote the initial and final draft of the abstract. BA reviewed the questionnaire and manuscript for submission and analyzed the collected data. AA was in charge of designing the questionnaire, as well as analyzing the collected data and writing the methodology, conclusions, and recommendations. TH was involved with the writing of the discussion, strengths, and limitations. All authors have critically reviewed and approved the final draft and are responsible for the content and similarity index of the manuscript.

\section{Acknowledgements}

We acknowledge all the volunteers who participated in the online survey to make this study possible. 


\section{References}

Al-Mohaithef, M., \& Padhi, B. K. (2020). Determinants of COVID-19 vaccine acceptance in Saudi Arabia: a web-based national survey. Journal of multidisciplinary healthcare, 13, 1657.

Assiri, A. (2021). Saudi Health Undersecretary: We aim to vaccinate 26 million people with the corona vaccine by the end of 2021 2021.. Saudi Arabia. Retrieved from https://bit.ly/35LBrPG

Benecke, O., \& DeYoung, S. (2019). Anti-Vaccine Decision-Making and Measles Resurgence in the United States. Global Pediatric Health, 6, 2333794X1986294. doi: $10.1177 / 2333794 \times 19862949$

Biswas, N., Mustapha, T., Khubchandani, J., \& Price, J. (2021). The Nature and Extent of COVID19 Vaccination Hesitancy in Healthcare Workers. Journal Of Community Health. doi: 10.1007/s10900-021-00984-3

Cordina, M., Lauri, M., \& Lauri, J. (2021). Attitudes towards COVID-19 vaccination, vaccine hesitancy and intention to take the vaccine. Pharmacy Practice, 19(1), 2317. doi: 10.18549/pharmpract.2021.1.2317

Coronavirus (COVID-19) Update: FDA Holds Advisory Committee Meeting to Discuss Authorization of COVID-19 Vaccine Candidate as Part of Agency's Review of Safety and Effectiveness Data. (2021). Retrieved 18 August 2021, from https://www.fda.gov/newsevents/press-announcements/coronavirus-covid-19-update-fda-holds-advisory-committeemeeting-discuss-authorization-covid-19

FDA Takes Additional Action in Fight Against COVID-19 By Issuing Emergency Use Authorization for Second COVID-19 Vaccine. (2021). Retrieved 18 August 2021, from https://www.fda.gov/news-events/press-announcements/fda-takes-additional-action-fightagainst-covid-19-issuing-emergency-use-authorization-second-covid 
Fine, P., Eames, K., \& Heymann, D. (2011). "Herd Immunity": A Rough Guide. Clinical Infectious Diseases, 52(7), 911-916. doi: 10.1093/cid/cir007

Fisher, K., Bloomstone, S., Walder, J., Crawford, S., Fouayzi, H., \& Mazor, K. (2020). Attitudes Toward a Potential SARS-CoV-2 Vaccine. Annals Of Internal Medicine, 173(12), 964-973. doi: $10.7326 / \mathrm{m} 20-3569$

Geldsetzer, P. (2020). Knowledge and Perceptions of COVID-19 Among the General Public in the United States and the United Kingdom: A Cross-sectional Online Survey. Annals Of Internal Medicine, 173(2), 157-160. doi: 10.7326/m20-0912

Gidengil, C., Parker, A., \& Zikmund-Fisher, B. (2012). Trends in Risk Perceptions and Vaccination Intentions: A Longitudinal Study of the First Year of the H1N1 Pandemic. American Journal Of Public Health, 102(4), 672-679. doi: 10.2105/ajph.2011.300407

Halpin, C., \& Reid, B. (2019). Attitudes and beliefs of healthcare workers about influenza vaccination. Nursing Older People, 31(2), 32-39. doi: 10.7748/nop.2019.e1154

Harrison, E., \& Wu, J. (2020). Vaccine confidence in the time of COVID-19. European Journal Of Epidemiology, 35(4), 325-330. doi: 10.1007/s10654-020-00634-3

Islam, M., Sarkar, T., Khan, S., Mostofa Kamal, A., Hasan, S., \& Kabir, A. et al. (2020). COVID19-Related Infodemic and Its Impact on Public Health: A Global Social Media Analysis. The American Journal Of Tropical Medicine And Hygiene, 103(4), 1621-1629. doi: 10.4269/ajtmh.200812

Kaur, S., \& Gupta, V. (2020). COVID-19 Vaccine: A comprehensive status report. Virus Research, 288, 198114. doi: 10.1016/j.virusres.2020.198114

Khubchandani, J., Saiki, D., \& Kandiah, J. (2020). Masks, Gloves, and the COVID-19 Pandemic: Rapid Assessment of Public Behaviors in the United States. Epidemiologia, 1(1), 16-22. doi: 10.3390/epidemiologia1010004 
Kim, H., Ahn, J., Atkinson, L., \& Kahlor, L. (2020). Effects of COVID-19 Misinformation on Information Seeking, Avoidance, and Processing: A Multicountry Comparative Study. Science Communication, 42(5), 586-615. doi: 10.1177/1075547020959670

Kreps, S., \& Kriner, D. (2020). Medical Misinformation in the COVID-19 Pandemic. SSRN Electronic Journal. doi: 10.2139/ssrn.3624510

Kwok, K., Lai, F., Wei, W., Wong, S., \& Tang, J. (2020). Herd immunity - estimating the level required to halt the COVID-19 epidemics in affected countries. Journal Of Infection, 80(6), e32e33. doi: 10.1016/j.jinf.2020.03.027

Larson, H., Clarke, R., Jarrett, C., Eckersberger, E., Levine, Z., Schulz, W., \& Paterson, P. (2018). Measuring trust in vaccination: A systematic review. Human Vaccines \& Immunotherapeutics, 14(7), 1599-1609. doi: 10.1080/21645515.2018.1459252

Lazarus, J., Ratzan, S., Palayew, A., Gostin, L., Larson, H., \& Rabin, K. et al. (2020). A global survey of potential acceptance of a COVID-19 vaccine. Nature Medicine, 27(2), 225-228. doi: $10.1038 / \mathrm{s} 41591-020-1124-9$

Loomba, S., de Figueiredo, A., Piatek, S., de Graaf, K., \& Larson, H. (2021). Author Correction: Measuring the impact of COVID-19 vaccine misinformation on vaccination intent in the UK and USA. Nature Human Behaviour, 5(3), 407-407. doi: 10.1038/s41562-021-01088-7

MacDonald, N. (2015). Vaccine hesitancy: Definition, scope and determinants. Vaccine, 33(34), 4161-4164. doi: 10.1016/j.vaccine.2015.04.036

Most Americans expect a COVID-19 vaccine within a year; $72 \%$ say they would get vaccinated. (2021). Retrieved 18 August 2021, from https://www.pewresearch.org/fact$\operatorname{tank} / 2020 / 05 / 21 /$ most-americans-expect-a-covid-19-vaccine-within-a-year-72-say-they-wouldget-vaccinated/

Murphy, J., Vallières, F., Bentall, R., Shevlin, M., McBride, O., Hartman, T., \& et al. (2021). Retrieved 18 August 2021, from 
Academic Journal of Research and Scientific Publishing | Vol 3 | Issue 29

Publication Date: 5-9-2021 ISSN: 2706-6495

https://www.researchgate.net/publication/342101254_Preparing_for_a_COVID-

19 vaccine_Identifying_and_psychologically_profiling those_who_are vaccine hesitant_or_re sistant_in_two_general_population_samples

NIH, COVID-19 Treatment Guidelines. (2021). Retrieved 18 August 2021, from https://www.covid19treatmentguidelines.nih.gov/about-the-guidelines/whats-new/

Østergaard, S., Schmidt, M., Horváth-Puhó, E., Thomsen, R., \& Sørensen, H. (2021). Thromboembolism and the Oxford-AstraZeneca COVID-19 vaccine: side-effect or coincidence?. The Lancet, 397(10283), 1441-1443. doi: 10.1016/s0140-6736(21)00762-5

Phadke, V., Bednarczyk, R., Salmon, D., \& Omer, S. (2016). Association Between Vaccine Refusal and Vaccine-Preventable Diseases in the United States. JAMA, 315(11), 1149. doi: 10.1001/jama.2016.1353

Polack, F., Thomas, S., Kitchin, N., Absalon, J., Gurtman, A., \& Lockhart, S. et al. (2020). Safety and Efficacy of the BNT162b2 mRNA Covid-19 Vaccine. New England Journal Of Medicine, 383(27), 2603-2615. doi: 10.1056/nejmoa2034577

Poland, G. (2020). Tortoises, hares, and vaccines: A cautionary note for SARS-CoV-2 vaccine development. Vaccine, 38(27), 4219-4220. doi: 10.1016/j.vaccine.2020.04.073

Qunaibi, E., Helmy, M., Basheti, I., \& Sultan, I. (2021). A high rate of COVID-19 vaccine hesitancy in a large-scale survey on Arabs. Elife, 10. doi: 10.7554/elife.68038

Reports and statistics. (2021). Retrieved 18 August 2021, from https://www.my.gov.sa/wps/portal/snp/aboutksa/saudiReportsAndStatistics\#header2_2

Ruiz, J., \& Bell, R. (2021). Predictors of intention to vaccinate against COVID-19: Results of a nationwide survey. Vaccine, 39(7), 1080-1086. doi: 10.1016/j.vaccine.2021.01.010

Salathé, M., \& Bonhoeffer, S. (2008). The effect of opinion clustering on disease outbreaks. Journal Of The Royal Society Interface, 5(29), 1505-1508. doi: 10.1098/rsif.2008.0271 
Sanche, S., Lin, Y., Xu, C., Romero-Severson, E., Hengartner, N., \& Ke, R. (2020). High Contagiousness and Rapid Spread of Severe Acute Respiratory Syndrome Coronavirus 2. Emerging Infectious Diseases, 26(7), 1470-1477. doi: 10.3201/eid2607.200282

Setbon, M., \& Raude, J. (2010). Factors in vaccination intention against the pandemic influenza A/H1N1. The European Journal Of Public Health, 20(5), 490-494. doi: 10.1093/eurpub/ckq054

Sherman, S., Smith, L., Sim, J., Amlôt, R., Cutts, M., \& Dasch, H. et al. (2020). COVID-19 vaccination intention in the UK: results from the COVID-19 vaccination acceptability study (CoVAccS), a nationally representative cross-sectional survey. Human Vaccines \& Immunotherapeutics, 17(6), 1612-1621. doi: 10.1080/21645515.2020.1846397

Szilagyi, P., Thomas, K., Shah, M., Vizueta, N., Cui, Y., Vangala, S., \& Kapteyn, A. (2021). National Trends in the US Public's Likelihood of Getting a COVID-19 Vaccine-April 1 to December 8, 2020. JAMA, 325(4), 396. doi: 10.1001/jama.2020.26419

Ten health issues WHO will tackle this year. (2021). Retrieved 18 August 2021, from https://www.who.int/news-room/spotlight/ten-threats-to-global-health-in-2019

UNICEF, G. (2021). COVID-19 Vaccination Hesitancy Survey Report January 2020. Retrieved 18 August 2021, from https://www.unicef.org/guyanasuriname/reports/covid-19-vaccinationhesitancy-survey-report-january-2020

Weinberg, G., \& Szilagyi, P. (2010). Vaccine Epidemiology: Efficacy, Effectiveness, and the Translational Research Roadmap. The Journal Of Infectious Diseases, 201(11), 1607-1610. doi: $10.1086 / 652404$

WHO Coronavirus Disease (COVID-19) Dashboard. (2020). Bangladesh Physiotherapy Journal, 10(1). doi: 10.46945/bpj.10.1.03.01

WHO Director-General's opening remarks at the media briefing on COVID-19 - 11 March 2020. (2021). Retrieved 18 August 2021, from https://www.who.int/director- 
general/speeches/detail/who-director-general-s-opening-remarks-at-the-media-briefing-on-covid19---11-march-2020

Wise, J. (2021). Covid-19: Rare immune response may cause clots after AstraZeneca vaccine, say researchers. BMJ, n954. doi: 10.1136/bmj.n954

Xiao, X., \& Wong, R. (2020). Vaccine hesitancy and perceived behavioral control: A metaanalysis. Vaccine, 38(33), 5131-5138. doi: 10.1016/j.vaccine.2020.04.076

Copyright (C) 2021 Dr. Roba Attar, Dr. Alaa Karkashan, Dr. Basma Abbas, Mr. Abdullatif Almarashi, Mr. Talat Hashem, AJRSP. This is an open-access article distributed under the terms of the Creative Commons Attribution License (CC BY NC).

Doi: doi.org/10.52132/Ajrsp.e.2021.292 